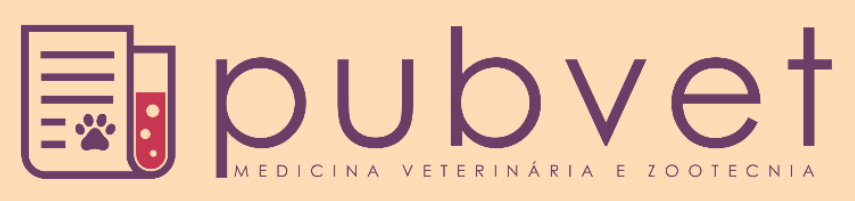

https://doi.org/10.31533/pubvet.v12n10a194.1-6

\title{
Projeto de intervenção de endoparasitoses em crianças cadastradas e acompanhadas pela Estratégia Saúde da Família no município de Tailândia, estado do Pará
}

\author{
Alliny Ferreira da Silva ${ }^{1 * \bullet}$, Geovania Maria da Silva Braga ${ }^{2} \bullet$ \\ ${ }^{1}$ Pós- Graduanda em Saúde Pública - UEMA. E-mail: allinyfg@hotmail.com. \\ ${ }^{2}$ Pós Doutora em Saúde Pública - FIOCRUZ. E-mail: geovaniacesi.uema@gmail.com \\ *Autor para correspondência
}

\begin{abstract}
RESUMO. Mais da metade da população mundial são afetados por parasitas intestinais causadas por inúmeros protozoários e helmintos. O objetivo desta pesquisa foi elaborar um plano de intervenção voltado para a prevenção de endoparasitoses em crianças de dois a cinco anos. Com a metodologia voltada ao estudo observacional na população assistida a partir da análise de prontuários, exames parasitológicos de fezes, sintomatologia relatada, condições sanitárias das residências e hábitos de higiene. Como resultados observou-se o índice elevado de crianças com decorrências positivas para endoparasitoses. Nota-se a necessidade da intervenção, através da educação em saúde/sanitária contínua, para que seja viabilizada a redução do índice de contaminação melhorando a informação e a qualidade de vida da comunidade.
\end{abstract}

Palavras chave: Estratégia saúde da família, helmintos, parasitos, protozoários

\section{Intervention project in endoparasitoses in children enrolled and accompanied by Family Health Strategy in the city of Thailand, State of Pará}

\begin{abstract}
More than half the world's population is affected by intestinal parasites caused by numerous protozoa and helminths. The objective of this research was to elaborate an intervention plan aimed at the prevention of endoparasitoses in children from two to five years. With the methodology focused on the observational study in the assisted population from the analysis of medical records, parasitological exams of stool, reported symptomatology, sanitary conditions of the residences and hygiene habits. As results, the high number of children with positive outcomes for endoparasitoses was observed. Regarding the conclusion, the need for intervention through continuous health/health education is noted, so that the reduction of the contamination index can be made possible by improving the information and the quality of life of the community.
\end{abstract}

Keywords: family health strategy, helminth, parasites, protozoan

\section{Projeto de intervenção em endoparasitoses nas crianças cadastradas e acompanhadas pela Estratégia Saúde da Família no município de Tailândia, estado do Pará}

RESUMEN. Más de la mitad de la población mundial son afectados por los parásitos intestinales causados por numerosos protozoos y helmintos. El objetivo de esta investigación fue elaborar un plan de intervención dirigido a la prevención de endoparásitos 
en niños de dos a cinco años. Con la metodología orientada al estudio observacional en la población asistida a partir del análisis de prontuarios, exámenes parasitológicos de heces, sintomatología relatada, condiciones sanitarias de las residencias y hábitos de higiene. Como resultados se observó el alto índice de niños con consecuencias positivas para endoparásitos. Es necesaria la intervención, a través de educación en salud/sanitaria continua, para que sea reducido el índice de contaminación y ampliada la comunicación informativa y consecuentemente mejorada la calidad de vida de la comunidad.

Palabras clave: Estrategia Salud de la familia, helmintos, parásitos, protozoarios

\section{Introdução}

As parasitoses intestinais causadas por inúmeros protozoários e helmintos afetam mais da metade da população mundial (WHO, 2003). Diversos estudos demonstram que a criança é a população alvo das infecções parasitárias, devido a hábitos de higiene, manejo inadequado dos alimentos, condições sanitárias e pela imaturidade do sistema imune. Nesta faixa etária essas parasitoses podem desencadear graves alterações fisiológicas (Heukelbach et al., 2003).

Os parasitas são vermes ou protozoários que se alojam no organismo, se alimentam de sangue ou do conteúdo intestinal e causam uma série de prejuízos, às vezes irremediáveis (Olivier, 2015). Cada tipo de parasitose tem a sua própria maneira de contaminação. A maioria delas pode ser evitada por medidas simples de higiene e limpeza (Olivier, 2015). O mesmo autor afirma que, a contaminação fecal-oral é a maneira mais simples e mais comum de infestação. Os ovos dos vermes e os cistos dos protozoários são eliminados do organismo pelas fezes. Quando os ovos ou os cistos são ingeridos com a água ou a alimentação, os parasitas ativos encontram condições propícias para o seu desenvolvimento e a infestação do novo organismo hospedeiro.

Os danos que os enteroparasitas podem causar a seus portadores incluem, entre outros agravos, a obstrução intestinal (A. lumbricoides), a desnutrição (A. lumbricoides e $T$. trichiura), anemia por deficiência de ferro (ancilostomídeos) e quadros de diarreia e de má absorção (E. histolytica e G. lamblia), sendo que as manifestações clínicas são usualmente proporcionais à carga parasitária albergada pelo indivíduo. Várias enteroparasitoses, incluindo infecções por Cryptosporidium parvum e Giardia lamblia (protozoários) são responsáveis por diarreia persistente com graves consequências, sobre o estado nutricional e desenvolvimento físico e mental, principalmente das crianças (Neves, 2005; Rey, 1991). O parasitismo é uma associação entre seres vivos com unilateralidade de benefícios, sendo o hospedeiro um dos associados e o prejudicado na associação, pois fornece o alimento e o abrigo ao parasita; assim, a parasitose é o estado de infecção cuja agressão repercute prejudicialmente sobre o hospedeiro (Neves, 2005). As parasitoses intestinais helmintíases e protozooses - representam a doença mais comum do globo terrestre. São endêmicas em países do terceiro mundo, onde se constituem problemas de Saúde Pública (Monteiro, 2011; WHO, 2003).

A Organização Mundial da Saúde (OMS) aponta alta frequência das doenças parasitárias na população mundial, estima-se que cerca de um bilhão de indivíduos em todo mundo alberguem o Ascaris lumbricoides, 795 estejam infestados por Trichuris trichiura e 740 milhões por ancilostomídeos (Ancylostoma duodenale e Necator americanos). Os parasitas intestinais estão entre os patógenos mais frequentemente encontrados em seres humanos, constituindo agravo importante à saúde. Dentre os helmintos, os mais frequentes são os nematelmintos Ascaris lumbricoides e Trichuris trichiura e os ancilostomídeos, sendo estes, os responsáveis pela maior carga de morbidade. Dentre os protozoários destacam-se Entamoeba histolytica e Giardia lamblia (WHO, 2003). A prevalência das enteroparasitoses é muito variada no país, ao redor do mundo e mesmo em comunidades de um mesmo município, pois o principal determinante são as condições de higiene e saneamento básico, bem como os níveis sócio econômicos e de escolaridade da população analisada (Andrade et al., 2010).

Os principais acometidos pelas doenças parasitárias são as crianças em idade escolar, por apresentarem hábitos de higiene, na maioria das vezes, inadequados e um sistema imunológico menos competente. Além disso, a conglomeração humana nas escolas e creches favorece a disseminação de agentes infeciosos. Por consequência, essas infestações podem comprometer o desenvolvimento físico, 
psicossomático e social das crianças, interferindo diretamente na qualidade de vida, gerando incapacidade funcional e deficiência no aprendizado (Morrone et al., 2004; Uchôa et al., 2001). A maior prevalência de parasitoses intestinais, entre crianças de regiões periféricas, reflete uma diferença na educação, cultura, hábitos alimentares e de higiene que as tornam mais suscetíveis à infecção (Santos et al, 1999). Os parasitas mais comumente encontrados nas crianças pré-escolares e escolares, em nosso meio, compreendem Giardia lamblia, Ascaris lumbricoides, Trichuris trichiura e Ancylostoma duodenalis, como a mais comum das 18 ancilostomíases (Manfroi et al., 2009).

De acordo com Olivier (2015) para se evitar este tipo de parasitose são importantes medidas profiláticas, como o tratamento adequado das fezes. O saneamento básico é fundamental. A utilização do vaso sanitário conectado à rede de esgoto tratado é a grande arma para o controle das parasitoses. Na ausência da rede de esgoto, a utilização de fossa cavada a boa distância da fonte de água potável, fechada e isolada, é solução satisfatória para quem reside em chácara ou no meio rural. Jamais evacuar no solo e, muito menos, utilizar as fezes para adubar plantações de alimentos. O tratamento adequado da água é outra importante medida profilática, onde a cloração da água potável é importante para a eliminação de bactérias patogênicas causadoras de infecções intestinais, como o vibrião colérico ou outras. No entanto, nem sempre é completamente suficiente para erradicar alguns ovos de parasitas ou cistos de protozoários mais resistentes. No caso, basta filtrar a água para torná-la potável. Se a água não for clorada na rede, você deve fervê-la ou adicionar cloro (que é fornecido gratuitamente na rede pública), na proporção de duas gotas para cada litro de água, e, depois, filtrá-la. Purificadores de água também são boa opção para substituir o processo de filtragem. Filtros instalados na rede da casa, fornecendo água filtrada para todos os cômodos, são práticos e possuem uma série de vantagens sobre os filtros tradicionais, desde que a sua manutenção não seja negligenciada. $\mathrm{O}$ uso exclusivo de água mineral engarrafada para consumo também é uma opção válida.

O tratamento adequado dos alimentos, levando em consideração todos os vegetais, como frutas, verduras, tubérculos e legumes, que devem ser cuidadosamente lavados em água corrente e clorados, para eliminarem-se eventuais ovos e cistos de parasitas presentes no solo.

As carnes bovinas e suínas são potencialmente transmissoras de parasitoses graves, como a teníase (solitária) e a neurocisticercose (Acevedo Nieto, 2015; Bürger et al., 2015). Toda carne deve ser inspecionada pelas autoridades da vigilância sanitária e o comércio clandestino deve ser desestimulado. A carne de porco, a carne de vaca $\mathrm{e}$, principalmente, as linguiças devem ser cozidas e nunca ingeridas malpassadas.

O diagnóstico é realizado por meio de exames parasitológicos de fezes, pelos métodos de sedimentação espontânea disponíveis na rotina, para a visualização dos ovos dos helmintos. Quando se objetiva a contagem de ovos por grama de fezes, que permite avaliar a intensidade da infecção, o método de diagnóstico recomendado é a técnica de Kato-Katz. Os métodos imunológicos, em geral, não são satisfatórios e não dispensam a coproscopia. Só têm indicação quando o exame de fezes for inconclusivo (Neves, 2005).

Segundo Ludwig et al. (1999), vários programas em Educação Sanitária têm sido dirigidos para o controle das parasitoses intestinais em diferentes países, mas, infelizmente, constatase um descompasso entre o êxito alcançado nos países mais desenvolvidos e aquele verificado nas economias mais pobres, em que vários fatores contribuem para a permanência deste quadro. De acordo com o Ministério da Saúde, as ações de saneamento ambiental buscam proteger e melhorar as condições de vida da população, mediante o abastecimento de água para consumo humano, esgotamento sanitário, coleta e disposição de resíduos sólidos e líquidos, melhoria de infraestrutura sanitária, instalações hidros sanitárias domiciliares, controle de vetores e de reservatórios de doenças transmissíveis. Contudo, a educação em saúde deve constituir parte essencial na promoção da saúde, na prevenção de doenças, como também contribuir para o tratamento precoce e eficaz das doenças, minimizando o sofrimento e a incapacidade.

A ação educativa na atenção primária estabelece-se a partir de programas determinados verticalmente, ou ligada às ações de promoção da saúde e prevenção da doença junto à comunidade, indivíduos ou grupos sociais, permeando densamente as atividades que os profissionais de saúde realizam no âmbito das unidades, no domicílio, em outras instituições e nos espaços comunitários (Witt, 2005). 
A finalidade desta pesquisa foi ordenar um plano de intercessão, voltada para a prevenção, precaução e vigilância em relação às endoparasitoses nas crianças de dois a cinco anos que estão cadastradas e acompanhadas pela Estratégia Saúde da Família no município de Tailândia, estado do Pará.

\section{Material e Métodos}

O estudo foi realizado no município de Tailândia, localizado no Nordeste no estado do Pará, a 260 quilômetros da capital do estado, Belém, entre os meses de julho a dezembro de 2017. O mesmo possui, de acordo com o Censo realizado em 2017 pelo IBGE, uma população de 103.321 habitantes, distribuídos em uma área de 4 $430,190 \mathrm{~km}^{2}$ de extensão territorial, com densidade de 23,32 hab. $/ \mathrm{km}^{2}$.

Apresenta um clima tropical, temperatura média de $27.0^{\circ} \mathrm{C}$, favorecendo, portanto, condições que propiciam a proliferação desses parasitas como declara Rey (1991) que favorecem amplamente a distribuição das parasitoses intestinais pelas regiões tropicais e temperadas do mundo, as parasitoses incidem mais intensamente nos lugares com clima quente e úmido, bem como onde as condições higiênicas da população são mais precárias.

Destaca-se para esta pesquisa o Bairro Novo, sendo observado que existem várias condições favoráveis a disseminação das endoparasitoses, como concentração populacional, baixas condições higiênicas e alimentares, o esgoto a céu aberto, a ausência de instalações sanitárias além das condições socioeconômicas precárias.

Para a coleta de dados foram escolhidas duas Estratégias Saúde da Família (ESF) localizada em uma unidade básica de saúde, no bairro citado acima, escolhido para o desenvolvimento deste estudo entre as famílias, com o intuito de propor um plano de intervenção de endoparasitoses, em crianças com a faixa etária de dois a cinco anos.

As equipes das ESF Bairro Novo I e II são compostas por enfermeiros, médicos, técnicos em enfermagem, agentes comunitários de saúde e o Núcleo de Apoio a Saúde da Família (NASF), que é composto pelo nutricionista, psicólogo, fisioterapeuta, assistente social e fonoaudiólogo.

Realizou-se um estudo observacional na população assistida a partir da análise de resultados de exames parasitológicos de fezes, sintomatologia relatada durante as consultas, visitas domiciliares possibilitando a avaliação das condições sanitárias das residências e hábitos de higiene.

Foram analisados 107 exames parasitológicos de fezes, em crianças na faixa etária entre dois (2) e cinco (5) anos, totalizando 79 resultados positivos e 28 negativos.

\section{Resultados}

As doenças parasitárias acometem geralmente as crianças em idade escolar, por apresentarem na maioria das vezes, hábitos de higiene inadequados e um sistema imunológico aquém do ajustado. Ainda, a conglomeração humana nas escolas e creches beneficia a dispersão de influentes infeciosos. Consequentemente, essas invasões podem afetar o desenvolvimento físico e social das crianças, intervindo abertamente na qualidade de vida das mesmas.

Durante as visitas domiciliares acompanhadas pelo enfermeiro e agente comunitário de saúde observaram-se condições precárias de saneamento, tratamento inadequado da água e em maioria higiene e manipulação de alimentos inadequados.

Tabela 1. Resultado do exame parasitológico de fezes em crianças de 2 a 5 anos, no Bairro Novo, município de Tailândia, estado do Pará, 2018.

\begin{tabular}{l|c|c|c}
\hline $\begin{array}{l}\text { Estratégia saúde } \\
\text { da família }\end{array}$ & $\begin{array}{c}\mathrm{N}^{\circ} \text { de exames } \\
\text { avaliados }\end{array}$ & $\begin{array}{c}\text { Resultados } \\
\text { positivos }\end{array}$ & $\begin{array}{c}\text { Resultados } \\
\text { negativos }\end{array}$ \\
\hline Bairro Novo I & 60 & 44 & 16 \\
\hline Bairro Novo II & 47 & 35 & 12 \\
\hline
\end{tabular}

\section{Discussão}

As endoparasitoses em crianças apresentam um índice elevado sendo considerado um sério agravo de saúde pública, pois interferem no organismo humano gerando alterações fisiológicas. Podemos observar que as variantes influenciam como, o tratamento inadequado da água, tal fato pode ser justificado, por uma população de baixa renda e conhecimento ineficaz considerar a água limpa e sem contaminação aquela que sai da torneira sem odor fétido, sem sabor e sem cor.

A distribuição de hipoclorito de sódio a 2,5\% é feita periodicamente nas residências de acordo com os relatos dos agentes comunitários de saúde, porém, observa-se a informação insuficiente dos usuários acerca do tratamento da água com o mesmo. Alguns domicílios possuem poços artesianos e os usuários lançam todo o conteúdo 
dentro dos mesmos, sendo o tratamento inoperante e desperdício ativo. Para purificar a água para consumo humano recomenda-se colocar duas gotas da solução para cada 1 litro de água e aguardar trinta minutos para o consumo.

Outro fator a ser considerado, é o manejo inadequado dos alimentos. Todos os alimentos devem ser criteriosamente higienizados na maneira correta, por exemplo, os vegetais necessitam ser lavados com água tratada e corrente com o objetivo de eliminar quaisquer agentes infecciosos. As carnes devem sempre bem cozidas para consumo e ser inspecionadas pela Vigilância Sanitária pois são transmissoras das verminoses mais graves.

A alta taxa de contaminação por parasitoses intestinais está ligada à falta de informação da população sobre as formas de contágio e profilaxia das mesmas, tendo em vista que, segundo Bagnato \& Renovato (2006), a maioria das parasitoses se dá por meio das condições de higiene ineficazes, somadas a falta de saneamento básico e más condições de moradia, fatores estes primordiais a serem discutidos nas práticas de Educação em Saúde/Sanitária continuadas, para que haja um resultado positivo em benefício da comunidade.

\section{Conclusão}

De acordo com as análises dos exames parasitológicos de fezes observou-se que, existe um elevado índice de endoparasitoses em crianças de dois (2) a cinco (5) anos cadastradas, nas duas Unidades de Estratégias da Saúde da Família escolhidas para este estudo, ocasionado por diversas e variadas causas tendo em conta que, as consequências dessas doenças representam danos à saúde coletiva humana, no município de Tailândia, estado do Pará.

\section{Consideração Final}

Durante as visitas domiciliares, consultas médicas e de enfermagem pode-se notar a necessidade da interação conjunta da equipe de saúde, com as famílias cadastradas para que melhore a informação diante da problemática, sugerindo então a educação em saúde com ações continuadas. Essas ações devem estar abordando os principais fatores determinantes para a contaminação como, tratamento adequado da água, higiene com os manipuladores de alimentos, higiene pessoal e mais além intensificar ações nas escolas.
Acredita-se que, a proposta de intervenção possibilite uma redução nos elevados índices de endoparasitoses em crianças nas Estratégias Saúde da Família Bairro Novo I e II, levando as informações necessárias e melhorando a qualidade de vida da comunidade assistida no município de Tailândia, estado do Pará.

\section{Referências Bibliográficas}

Acevedo Nieto, C. E. 2015. Complexo teníasecisticercose em assentamentos da Reforma Agrária do estado de Minas Gerais, Brasil. Master of Science, Universidade Federal de Viçosa, Viçosa.

Andrade, E. C., Leite, I. C. G., Oliveira Rodrigues, V., \& Cesca, M. G. 2010. Parasitoses intestinais: uma revisão sobre os seus aspectos sociais, epidemiológicos, clínicos e terapêuticos. Revista de APS, 13(2), 231-240.

Bagnato, M. H. S., \& Renovato, R. D. 2006. Práticas educativas em saúde: um território de saber, poder e produção de identidades. In R. A. Deitos \& R. M. Rodrigues (Eds.), Estado, desenvolvimento, democracia \& políticas sociais (pp. 87-104). Cascavel, Paraná: EDUNIOESTE.

Bürger, K. P., Buzza, P. L. T., Neto, E. B., dos Santos, L. R., Rossi, G. A. M., \& Martins, A. M. C. V. 2015. Complexo teniose-cisticercose: ocorrência em abatedouro de bovinos e conhecimento de estudantes do ensino médio e consumidores no Estado de São Paulo, Brasil. Revista Brasileira de Ciência Veterinária, 22(1), 1-10.

Heukelbach, J., Oliveira, F. A. S., \& Feldmeier, H. 2003. Ectoparasitoses e saúde pública no Brasil: desafios para controle Ecoparasitoses and public health in Brazil: challenges for control. Caderno de Saúde Pública, 19(5), 1535-1540.

Ludwig, K. M., Frei, F., Alvares Filho, F., \& Ribeiro-Paes, J. T. 1999. Correlação entre condições de saneamento básico e parasitoses intestinais na população de Assis, Estado de São Paulo. Revista da Sociedade Brasileira de Medicina Tropical, 32(5), 547-555.

Manfroi, A., Stein, A. T., \& Castro Filho, E. D. (2009). Abordagem das parasitoses intestinais mais prevalentes na infância. Sociedade Brasileira de Medicina de Família e Comunidade, 8, 1-26.

Monteiro, S. G. 2011. Parasitologia na medicina veterinária (Vol. 1). São Paulo: Roca. 
Morrone, F. B., Carneiro, J. A., Reis, C., Cardozo, C. M., Ubal, C., \& De Carli, G. A. 2004. Study of enteroparasites infection frequency and chemotherapeutic agents used in pediatric patients in a community living in Porto Alegre, RS, Brazil. Revista do Instituto de Medicina Tropical de São Paulo, 46(2), 77-80.

Neves, D. P. 2005. Parasitologia humana. São Paulo, Brasil: Editora Atheneu.

Olivier, C. E. 2015. Puericultura; preparando o seu filho para o futuro. São Paulo, Brasil: Nonas edições acadêmicas.

Rey, L. P. E. G., Rio de Janeiro. (1991). Parasitologia. Rio de Janeiro, Brasil: Editora Guanabara.

Uchôa, C., Lobo, A. G. B., Bastos, O. M. P., \& Matos, A. D. 2001. Parasitoses intestinais: prevalência em creches comunitárias da cidade de Niterói, Rio de Janeiro-Brasil. Revista do Instituto Adolfo Lutz, 60, 97-101.
WHO. 2003. World Health Organization. Diet, nutrition and the prevention of chronic diseases. Report of a joint WHO/FAO expert consultation. WHO technical report series, 916.

Witt, R. R. 2005. Competências da enfermeira na atenção básica: contribuição à construção das funções essenciais de saúde pública. $\mathrm{PhD}$, Universidade de São Paulo, Ribeirão Preto, São Paulo.

Recebido: 17 Julho, 2018

Aprovado: 28 Agosto, 2018

Publicado: 26 Outubro, 2018

Licenciamento: Este artigo é publicado na modalidade Acesso Aberto sob a licença Creative Commons Atribuição 4.0 (CC-BY 4.0), a qual permite uso irrestrito, distribuição, reprodução em qualquer meio, desde que o autor e a fonte sejam devidamente creditados. 